\title{
Vorschlag einer Qualitätssicherungsmethode für geogen belasteten Bodenaushub
}

\author{
Martin Wellacher · Markus Brechlmacher · Franz Poschacher · Roland Pomberger
}

Online publiziert: 11. November 2019

(C) Der/die Autor(en) 2019

Zusammenfassung Geogene Belastungen von Metallen gibt es in vielen Böden, auch in der Steiermark. Bodenaushub, der verwertet werden soll, muss gesetzlich festgelegte Grenzwerte, u. a. für Metalle, unterschreiten. Über solche Grenzwerte geogen belasteter Bodenaushub ist in Österreich zur Deponierung bestimmt. Es ist jedoch Stand der Wissenschaft und Forschung, dass auch in grenzwertüberschreitenden Gehalten vorkommende Metalle nicht mobil sein müssen und so auch keine toxische Wirkung hätten.

Die vorgestellte Entwicklung betrifft Böden aus geogen mit Metallen belasteten Regionen, wie sie in der Steiermark häufig vorkommen. Für von dort stammenden Bodenaushub wurde eine Qualitätssicherungsmethode entwickelt, mit der festgestellt werden kann, ob dieser unbedenklich zur Verwertung geeignet ist oder bedenklich ist und daher nur deponiert werden darf.

Im Zuge der vorgeschlagenen Methode wurden drei Fragen behandelt und beantwortet: 1) Ist die Belastung eines Bodens geogenen oder anthropogenen Ursprungs? 2) Wie mobil sind die betrachteten Metalle im Bodenaushub hinsichtlich ihres Transfers vom Boden in Pflanzen? 3) Wie mobil sind die betrachteten Metalle hinsichtlich ihres Transfers vom Boden ins Grundwasser? Die Methode besteht dementsprechend aus drei Teilen, einer Methode

Mag. Dr. M. Wellacher $(\bowtie)$.

M. Brechlmacher .

Univ.-Prof. DI Dr. R. Pomberger

Lehrstuhl für

Abfallverwertungstechnik

und Abfallwirtschaft,

Montanuniversität Leoben,

Franz-Josef-Straße 18, 8700 Leoben,

Österreich

martin.wellacher@unileoben.ac.at

F. Poschacher

Poschacher Kompost,

Hubertusgasse 8, 8714 Kraubath/M,

Österreich zur Unterscheidung geogener von anthropogenen Metallbelastungen, einem Kleingefäßtest und einem Großgefäßtest.

Schlüsselwörter Boden · Metall · Geogen · Bodenaushub

Proposal for a quality assurance method for excavated soil with geogenic metal contamination

Abstract Geogenic contaminations of metals in soils exist in many regions such as in the Austrian province of Styria. Excavated soil dedicated for recycling must under-run legal limit values for metals as well. Material above those limits must be landfilled. It is state of the art of science and technology that even exceeding metal concentrations do not need to be mobile thus are not toxic.

The proposed development concerns soils from with metals geogenicly contaminated regions, as they are typical for Styria. A quality assurance method was developed for excavated soil from these areas, by which it can be determined whether the recycling is unobjectionable and can be recommended for recycling or worrying thus should be landfilled only.

In the course of the proposed method three questions are discussed and answered: 1) Is the origin of the contamination geogenic or anthropogenic? 2) How is the degree of mobility of the concerned metals with regard to the transfer from soil to plants? 3) How is the degree of mobility of the concerned metals with regard to the transfer from soil to groundwater? Thus the method consists of three parts, a method to distinguish geogenic from anthropogenic metal contaminations, a small vessel test and a big vessel test.

Keywords Soil · Metal · Geogenic · Excavated soil

\section{Einleitung}

Die Verwertung von Aushubmaterialien in Österreich ist eine wichtige, aber noch $\mathrm{zu}$ wenig entwickelte abfallwirtschaftliche Maßnahme für diesen größten aller Abfallströme. Sie wird durch fachliche, wirtschaftliche und gesetzliche Rahmenbedingungen ermöglicht.

Das Aufkommen an Aushubmaterialien und Böden in Österreich im Jahr 2017 betrug 32,3 Mio. t, wovon nur $23 \%$ verwertet, der Rest aber deponiert wurde (BMNT 2019). Die Verwertungsquote in Österreich sinkt in den letzten Jahren (Brechlmacher und Wellacher 2018). Das Aufkommen an Aushubmaterialien im Bundesland Steiermark betrug 2015 2,4 Mio. t, die Verwertungsquote ist nicht bekannt. Für die Steiermark sind zahlreiche Standorte mit geogenen Belastungen mit Metallen beschrieben (z.B. Reinhofer et al. 2004). Beispielsweise ist die natürliche geogene Ni-Belastung im Bereich der MurMürz-Furche langjährig bekannt (Amt der Steiermärkischen Landesregierung 2004). Ni-Gehalte in biogenen Abfällen von $100 \mathrm{mg} / \mathrm{kg}$ TM sind nicht ungewöhnlich. Es wird angenommen, dass die derzeitigen Marktgegebenheiten das Deponieren von Aushubmaterialien gegenüber der Verwertung bevorzugen (Wellacher et al. 2018).

Bei einer Verwertung werden die Funktionen des ursprünglichen Bodens größtenteils erhalten, während sie bei der Deponierung in überwiegendem $\mathrm{Maß}$ dauerhaft verloren sind. Diese Bodenfunktionen haben mittel- und langfristig lebenserhaltende Wirkungen auf Mensch und Umwelt und sind daher von besonderer Bedeutung. Es sind dies Lebensraumfunktionen, Regelungsfunktionen (Böden sind Speicher-, Filter-, Puffer- und Transformationssysteme) und Nutzungsfunktionen (u.a. die Eignung für land- und forstwirtschaftliche Nutzung, Wald- und Waldfunktionsstandorte in urbanen Bereichen, Parks, Gärten, Spiel- und Sportplätze) (Drobnik et al. 2018). 
In Österreich betrug die auf Aushubtätigkeiten folgende Bodenversiegelung $20162200 \mathrm{~km}^{2}$. Dies ist ein Umweltproblem, für das eine Lösung zu finden ist. Im Zuge von Nutzungsänderungen wurden weitere $3347 \mathrm{~km}^{2}$ in Anspruch genommen (Umweltbundesamt 2017). Nach Abschluss der Bautätigkeiten stellen sie den Markt für Rekultivierungsmaßnahmen dar. Die negativen Auswirkungen der Bodenzerstörung können durch die Verwertung von Bodenaushub z. B. für eben diese Rekultivierungsmaßnahmen gemildert werden.

Die für Bodenaushub zur Verwertung und Deponierung gültigen Grenzwerte finden sich im regelmäßig veröffentlichten Bundesabfallwirtschaftsplan (BAWP), zuletzt 2017 (BMLFUW 2017). Der BAWP gilt als ein objektiviertes standardisiertes Gutachten zum Stand der Technik, und ist kein Gesetz. Diese Grenzwerte wurden auch in andere Regelungen übernommen, z.B. in die Richtlinie „Gewässerschutz an Straßen“ des Bundesministeriums für Verkehr, Innovation und Technologie (BMVIT 2011) und haben eine weit verbreitete Gültigkeit. Es ist möglich, vom BAWP abweichend mittels spezifiziertem Gutachten und in Absprache mit der Behörde unbedenkliche Materialien in die Verwertung zu führen.

In einigen geogen belasteten Böden gibt es z.B. nur ein Metall, welches den Grenzwert des BAWP 2017 überschreitet. Das Material kann dann zwar bei allen anderen grenzwertrelevanten Parametern die Vorgaben des BAWP 2017 erfüllen, muss aber trotzdem deponiert werden. Es ist Stand der Wissenschaft und Forschung, dass auch in grenzwertüberschreitenden Gehalten vorkommende Metalle nicht mobil sein müssen und so auch keine toxische Wirkung hätten (Tessier et al. 1979; Fang et al. 2016).

Der BAWP 2017 definiert zur Verwertung von Bodenaushub die Qualitätsklasse „Bodenaushub mit geogener Belastung“ (oder BA mit der Fußnote 6) ohne für den Begriff "geogen“ eine Methodik zur Unterscheidung von einer anthropogenen Belastung anzubieten. Es handelt sich dabei um die Bodenaushubklasse mit den höchsten Grenzwerten, die nur in Gebieten vergleichbarer Belastungssituation verwertet werden darf. Die Unterscheidung von geogen und anthropogen ist daher bedeutsam. Der Hintergrund dafür ist, dass fachlich postuliert wird, Metalle aus geogenen Quellen seien weniger mobil.
Bei entsprechender technischer Eignung und bei Einhaltung der jeweiligen Qualitätskriterien darf Aushubmaterial als Rohstoff für die Bodenrekultivierung, die Untergrundverfüllung, zur Herstellung von Komposterden oder sonstiger Erden, als Recycling-Baustoff, zur Kompostierung oder für industrielle Anwendungen verwendet werden (BMLFUW 2017).

Bestimmte Aushubmaterialien können durch Sieben alleine zu einem verkaufsfähigen Rekultivierungssubstrat oder einem anderen Produkt aufbereitet werden. Auch eine direkte Verwertung von nicht aufbereitetem Bodenaushub ist denkbar. Für die Herstellung einer Komposterde werden Bodenaushub mit Kompost und gegebenenfalls anderen Komponenten, z. B. Sand oder Ziegelsplitt, gemischt (ÖWAV 2014).

Ziel dieses Beitrages ist es, die im Projekt ReSoil entwickelte Qualitätssicherungsmethode für Bodenaushub mit grenzwertüberschreitenden geogenen Metallbelastungen darzustellen und zu begründen.

\section{Material und Methoden}

Für die Auswahl geeigneter Böden wurden 11 Grundstücke in der Steiermark mit bekannt hohen Metallgehalten herangezogen. Die Festlegung dieser Grundstücke erfolgte auf Basis des Landesumweltinformationssystems Steiermark (LUIS) (Amt der Steiermärkischen Landesregierung 2017a). Dieses System erlaubt in Kombination mit dem Geographischen Informationssystem Steiermark (GIS) (Amt der Steiermärkischen Landesregierung 2017b) eine Abfrage eines landesweiten Untersuchungsrasters in Bezug auf Bodenschutz (siehe auch LwVzSt 1999). Ein unbelasteter Standort, dessen Nutzung über Jahrzehnte bekannt war, wurde als Referenzprobe verwendet (L3).

Bodensubstrate sind Mischungen von Bodenaushub und anderen Komponenten zur Herstellung von verkaufsfähigen Produkten für die Rekultivierung bzw. Sanierung. Die Mischungen werden spezifisch für die jeweilige Anwendung hergestellt, z.B. als Gartenerde oder als Sickerrasensubstrat. Bei Bedarf wird das Substrat vor Auslieferung noch gesiebt.

Die Probenahme der Bodensubstrate erfolgte nach ÖNORM S2127 (ASI 2011). Dabei wurden 10 bis 40 Stichproben zu Sammelproben vereinigt.
Die untersuchten Metalle waren As, $\mathrm{Cd}, \mathrm{Cr}, \mathrm{Cu}, \mathrm{Hg}$, Ni und $\mathrm{Pb}$. Die Metalle wurden als Gesamtgehalte mit Königswasser-Aufschluss (Aqua regia, A.r.) und als Eluatgehalte bestimmt (Bundesgesetz 2008).

\subsection{Methode zur Unterscheidung von geogenen und anthropogenen Metallbelastungen in Böden}

Für die Probenahme mittels Pürckhauer-Methode wurde der Hohlmeißelbohrer an den ausgewählten Grundstücken an 25 Stellen mit $5 \mathrm{~m}$ Reihenabstand $1 \mathrm{~m}$ tief in den Boden eingeschlagen. Für die Probenahme wurden die untersten $25 \mathrm{~cm}$ des Pürckhauers (ca. 50 bis $100 \mathrm{~cm}$ Tiefe) jeweils zu einer Sammelprobe je Lokalität vereint und als „Untere Bodenschicht“ benannt. Die daran anschließenden $25 \mathrm{~cm}$ (ca. 5 bis $50 \mathrm{~cm}$ Tiefe) wurden ebenfalls zur Sammelprobe „Obere Bodenschicht" vereinigt. Die obere Pflanzenschicht 0 bis $5 \mathrm{~cm}$ wurde entfernt. Die Probennahme entsprach der ÖNORM L1056 (ASI 2004).

Eine unbedenkliche Verwertung von Aushubmaterialien im ersten Teil wurde festgelegt, wenn der Metallgehalt geogener und nicht anthropogener Natur ist. Geogen ist ein Metallgehalt wenn

1. die Gehalte der unteren Bodenschicht mindestens $20 \%$ höher oder gleich hoch wie jene der oberen Bodenschicht sind und

2. die Gehalte der Fraktion $>2 \mathrm{~mm}$ mindestens $20 \%$ höher oder gleich hoch wie jene der Fraktion $<2 \mathrm{~mm}$ sind.

Nur wenn beide Aussagen zutreffen, wird der Metallgehalt als geogen eingestuft.

\subsection{Kleingefäßversuch}

Die Grundüberlegung des Kleingefäßtests ist, dass ein Material dann unbedenklich ist, wenn von ihm keine toxischen Wirkungen über einen bedenklichen Transfer in Futter- oder Lebensmittelpflanzen ausgehen. Da$\mathrm{zu}$ wurden die gültigen Grenzwerte der europäischen Lebensverordnung (Europäische Union 2006) und der europäischen Futtermittelrichtlinie (Europäische Union 2002) herangezogen. Wo keine Grenzwerte vorlagen, bei Ni und Cr., wurde auf Literaturwerte von auf unbelasteten Böden kultivierten Pflanzen gleicher oder ähnlicher Art 


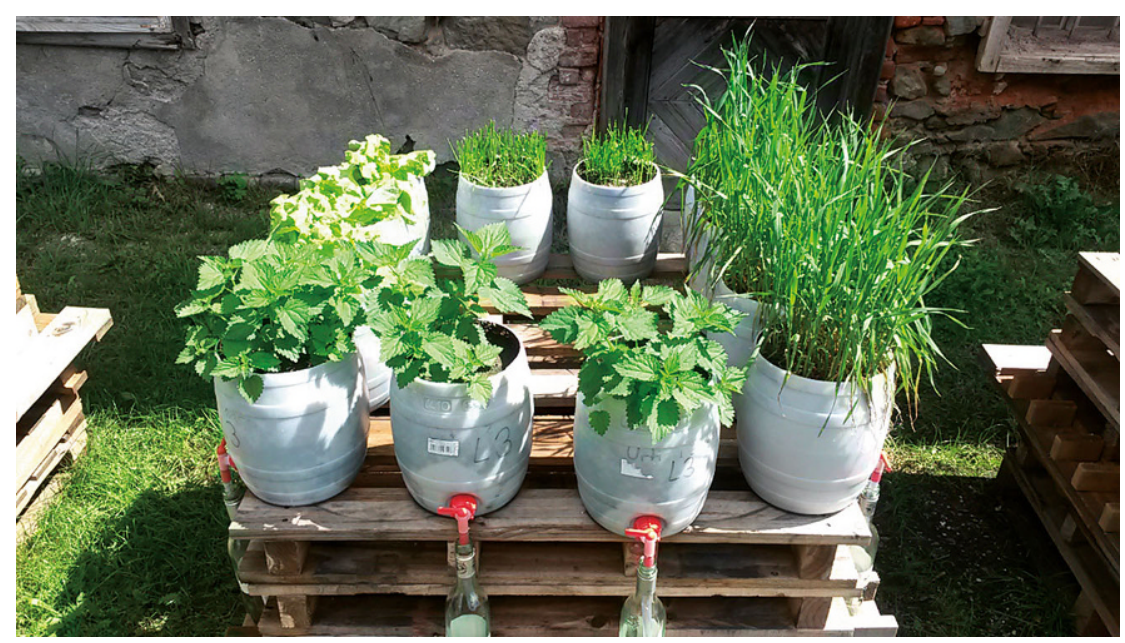

Abb. 1 Versuchsansätze bei der Entwicklung des Kleingefäßtests mit vier Pflanzenspezies in je drei Wiederholungen für jedes Testmaterial

zurückgegriffen (Kabata-Pendias und Pendias 2001).

Für die Klein- bzw. Großgefäßversuche wurden 20 bis 25 Einzelproben je untersuchtem Grundstück in einem definierten Raster mit $5 \mathrm{~m}$ Reihenabstand durch Öffnen bzw. Abheben der Grasoberfläche entnommen. Hierzu wurden je Probenahmestelle (ca. $0,5 \mathrm{~m} \times 0,5 \mathrm{~m}$ ) Bodenmaterial von $-10 \mathrm{~cm}$ bis zu einer Tiefe von $-30 \mathrm{~cm}$ entnommen. Die Einzelproben wurden zu je einer Sammelprobe pro Lokalität (200 bis $800 \mathrm{l}$ ) vereinigt. Die Probennahme entsprach der ÖNORM L1056 (ASI 2004).

Die Metallgehalte der Pflanzen im Kleingefäßtest wurden nach Austrian Standards Institute (2002, 2006) bestimmt.

Als Kriterien für die Validierung des Tests sollten die besten Zeigerpflanzen gefunden und ihr Pflanzenwuchs (Blattlänge und Erntegewicht) bestimmt werden. Die getesteten Pflanzenspezies waren Hafer (Avena sativa), Kopfsalat (Lactuca sativa), Italienisches Raygras (Lolium multiflorum) und Große Brennessel (Urtica dioica).

Die verwendeten Gefäße waren Kunststoffässer mit Ablauf, die mit ca. 101 Substrat befüllt wurden. Die Kulturdauer bis zur Ernte betrug 70 bis $120 \mathrm{~d}$.

Es wurden fünf Bodenmaterialien getestet, davon ein Standardboden ohne Belastung (L3). Der Boden L5N1 wurde mit einer $\mathrm{NiSO}_{4}$-Lösung durchmischt, die im Boden einen Ni-Gehalt von $514 \mathrm{mg} / \mathrm{kg}$ TM ergab.
Die Kultivierung erfolgte unter freiem Himmel am Standort Kraubath/M. (Abb. 1).

\subsection{Großgefäßversuch}

Die Grundüberlegung des Großgefäßtests ist, dass ein Material dann unbedenklich ist, wenn von ihm keine toxischen Wirkungen durch einen Transfer in Grund- und damit Trinkwasser ausgehen. Dazu wurden die gültigen Werte der österreichischen Trinkwasserverordnung herangezogen (Bundesgesetz 2001).

Von einem Intermediate Bulk Container (IBC, 1000l, verschließbarer Ablauf) wurde der Oberteil abgeschnitten, sodass eine freie Fläche von etwa $1,2 \mathrm{~m}^{2}$ entstand. Der IBC wurde in vier Schichten befüllt, zuunterst mit zwei Schich-

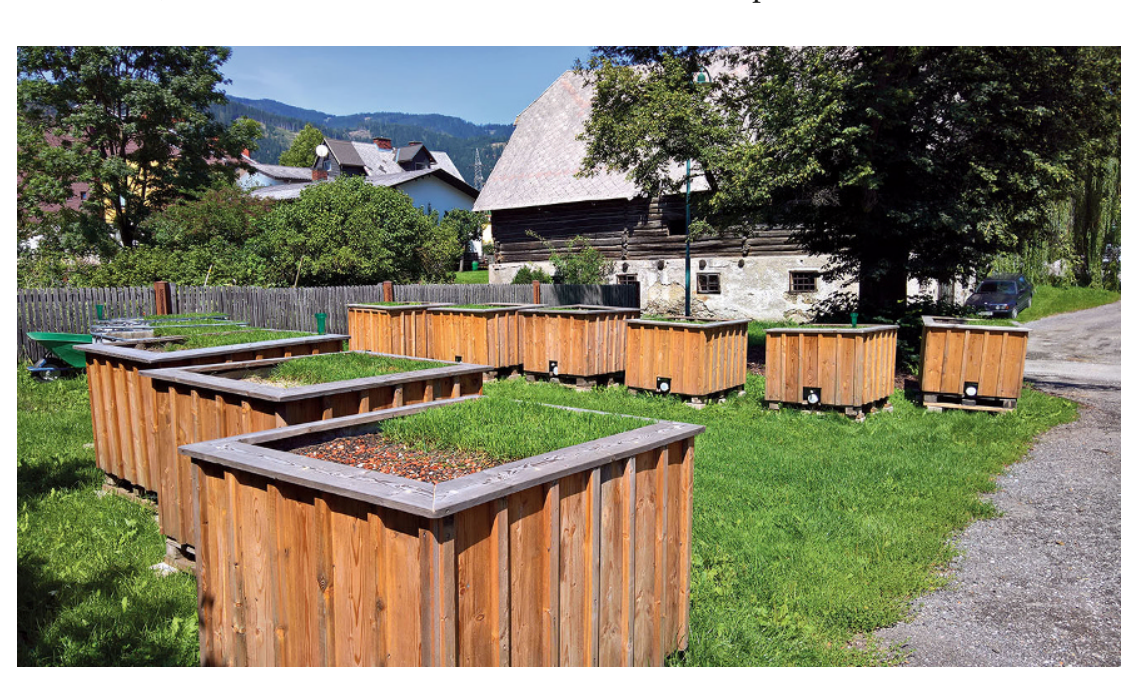

Abb. 3 Versuchsansätze zur Entwicklung des Großgefäßtests

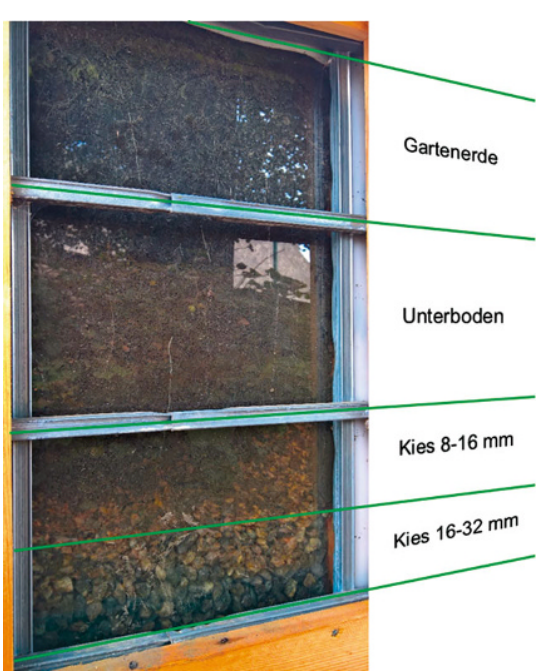

Abb. 2 Schichtung im Großgefäß an einem Sichtfenster eines IBC

ten 2-fach gewaschenem Kies, zuerst 16 bis $32 \mathrm{~mm}$ Korngröße und darüber 8 bis $16 \mathrm{~mm}$. Als dritte Schicht wurde Unterboden L3 $<10 \mathrm{~mm}$ Korngröße ( -25 bis $-50 \mathrm{~cm}$ Bodentiefe) ca. $25 \mathrm{~cm}$ hoch aufgetragen. Als oberste Schicht wurden $30 \mathrm{~cm}$ des $\mathrm{zu}$ untersuchenden Bodensubstraes eingefüllt (Abb. 2).

Der Versuchsstand wurde am Standort Kraubath/Mur aufgebaut (Abb. 3).

Für die Versuche wurden fünf unterschiedliche Bodensubstrate herangezogen. Zusätzlich wurde eine Vergleichsprobe mit dem Standardboden L3 befüllt ( -5 bis $25 \mathrm{~cm}$ Bodentiefe). Eine Grasmischung („Quickplay“ der Austrosaat, Österreichische Samenzuchtu. Handels-Aktiengesellschaft) wurde ausgesät und gewartet, bis ein bodendeckender Bewuchs vorhanden war. $\mathrm{Zu}$ diesem Zeitpunkt wurden die Boden- 
ventile der Gefäße dicht verschlossen. Nach drei Wochen und regelmäßigem sowie abschließenden Bewässern wurde das entstandene Sickerwasser in seinem Volumen erfasst sowie beprobt und auf seinen Metallgehalt analysiert.

Die Analysen der Metalle in den Sickerwässern erfolgten gemäß ÖNORM EN ISO 17294-2 (ASI 2016).

\section{Ergebnisse}

Die Qualitätssicherungsmethode konnte in allen drei Teilen entwickelt werden. Mit dieser Methode ist eine Prüfung auf Unbedenklichkeit von geogenen grenzwertüberschreitenden Belastungen mit Metallen möglich.

Eine unbedenkliche Verwertung von Aushubmaterialien mit geogener Belastung liegt vor, wenn

1. die Gehalte der unteren Bodenschicht mindestens $20 \%$ höher oder gleich hoch wie jene der oberen Bodenschicht sind und

2. die Gehalte der Fraktion $>2 \mathrm{~mm}$ (der oberen Bodenschicht) mindestens $20 \%$ höher oder gleich hoch wie jene der Fraktion $<2 \mathrm{~mm}$ sind und

3. Salatpflanzen der Spezies Lactuca sativa im Kleingefäßtest in ihren $\mathrm{Ge}$ samtgehalten keine Überschreitung von Grenzwerten für Lebensmittel zeigen und

4. Sickerwasser aus dem Großgefäßtest die Grenzwerte für Trinkwasser nicht überschreitet (Bundesgesetz 2001).

Hierzu sind die ersten beiden Untersuchungen am nativen Boden vor dem Aushub durchzuführen, während die letzten beiden nach dem Aushub am
Tab. 1 Übersicht zur Beurteilung der 12 untersuchten Böden auf anthropogen (a) oder geogen (g) für sieben Metalle

\begin{tabular}{|l|l|l|l|l|l|l|l|l|l|l|l|l|l|}
\hline Metalle/Boden & L1 & L2 & L3 & L4 & L5 & L7 & L8 & L9 & L11 & L12 & L13 & L14 \\
\hline As oben/unten & g & a & - & - & - & a & g & g & g & a & - & - \\
\hline As $</>2 \mathrm{~mm}$ & - & g & - & - & - & g & - & g & a & a & - & - \\
\hline Cd oben/unten & g & a & - & - & g & - & - & - & - & - & - & - \\
\hline $\mathrm{Cd}</>2 \mathrm{~mm}$ & $\mathrm{~g}$ & $\mathbf{a}$ & - & - & $\mathrm{g}$ & - & - & - & - & - & - & - \\
\hline $\mathrm{Cr}$ oben/unten & - & - & - & - & - & - & - & $\mathrm{g}$ & - & - & - & $\mathrm{g}$ \\
\hline $\mathrm{Cr}</>2 \mathrm{~mm}$ & - & - & - & - & - & - & - & - & - & - & - & - \\
\hline $\mathrm{Cu}$ oben/unten & $\mathrm{g}$ & $\mathbf{a}$ & - & - & - & $\mathrm{g}$ & - & $\mathbf{a}$ & $\mathbf{a}$ & $\mathrm{g}$ & - & - \\
\hline $\mathrm{Cu}</>2 \mathrm{~mm}$ & - & - & - & - & - & $\mathbf{a}$ & - & $\mathrm{g}$ & $\mathbf{a}$ & $\mathbf{a}$ & - & - \\
\hline $\mathrm{Hg}$ oben/unten & $\mathrm{g}$ & - & - & - & $\mathbf{a}$ & - & - & - & $\mathrm{g}$ & $\mathrm{g}$ & - & - \\
\hline $\mathrm{Hg}</>2 \mathrm{~mm}$ & $\mathrm{~g}$ & $\mathbf{a}$ & - & - & $\mathrm{g}$ & - & - & - & $\mathbf{a}$ & $\mathbf{a}$ & - & - \\
\hline $\mathrm{Ni}$ oben/unten & - & - & - & - & - & - & - & $\mathrm{g}$ & - & - & $\mathbf{a}$ & $\mathrm{g}$ \\
\hline $\mathrm{Ni}</>2 \mathrm{~mm}$ & - & - & - & - & - & - & - & - & - & - & $\mathrm{g}$ & $\mathrm{g}$ \\
\hline $\mathrm{Pb}$ oben/unten & $\mathrm{g}$ & $\mathbf{a}$ & - & - & $\mathbf{a}$ & $\mathrm{g}$ & - & - & - & $\mathbf{a}$ & - & - \\
\hline $\mathrm{Pb}</>2 \mathrm{~mm}$ & $\mathbf{a}$ & $\mathrm{g}$ & - & - & $\mathrm{g}$ & $\mathbf{a}$ & - & - & - & $\mathbf{a}$ & - & - \\
\hline
\end{tabular}

Bodenaushub und den daraus hergestellten Produkten stattfinden.

\subsection{Unterscheidung geogener und anthropogener Metallbelastung}

Die 12 untersuchten Böden konnten mit der vorliegenden Methode gut in zwei unbelastete, zwei geogen belastete und acht anthropogen belastete unterschieden werden. Insgesamt waren somit 84 Zuordnungen zu treffen. Einige der anthropogen belasteten Materialien enthielten mehrere Metallbelastungen, von denen zumindest eine bei den Untersuchungen als anthropogen identifiziert wurde. Zwei Böden konnten hinsichtlich der Metalle, die in grenzwertüberschreitenden Konzentrationen vorlagen, als geogen beurteilt werden (Tab. 1).
Beispielhaft seien die Ergebnisse für $\mathrm{Hg}$ ausgeführt. Drei der untersuchten Bodensubstrate waren über dem niedrigsten BAWP 2017 Grenzwert von $0,5 \mathrm{mg} / \mathrm{kg}$ TM. Davon konnten ein Boden (L5) als anthropogen und zwei Böden (L11, L12) als geogen eingestuft werden (Abb. 4).

\subsection{Kleingefäßversuch}

Der Versuchsaufbau des Kleingefäßtests war geeignet, um einen bedenklichen Transfer von Metallbelastungen in Bodenaushub oder daraus hergestellten Produkten in verschiedene Pflanzenarten zu erkennen und von einem unbedenklichen Transfer zu unterscheiden.

Die Wachstumsverläufe der vier Testpflanzen zeigten teilweise signifikante Unterschiede zwischen Referenzprobe
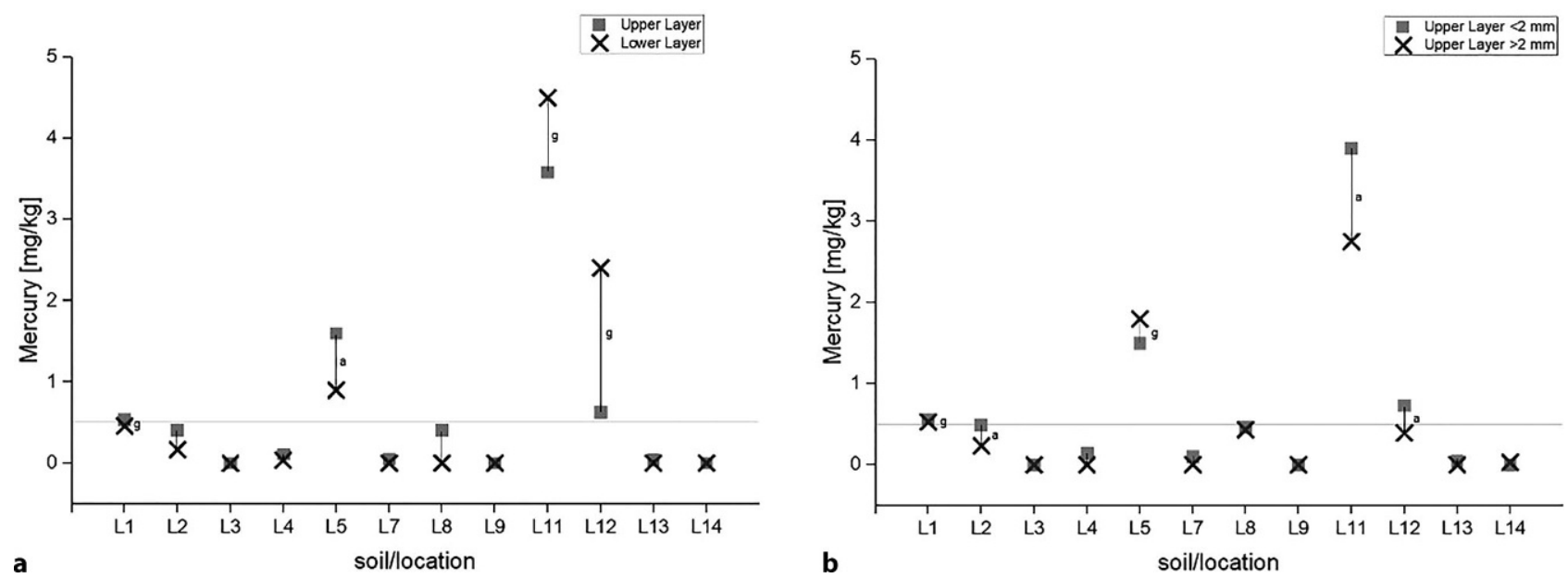

Abb. 4 Unterscheidung von 12 Bodenmaterialien hinsichtlich geogener (g) und anthropogener (a) Belastung mit Hg (die waagrechte Linie gibt den untersten BAWP 2017 Grenzwert für $\mathrm{Hg}$ von 0,5 mg/kg TM wieder) 


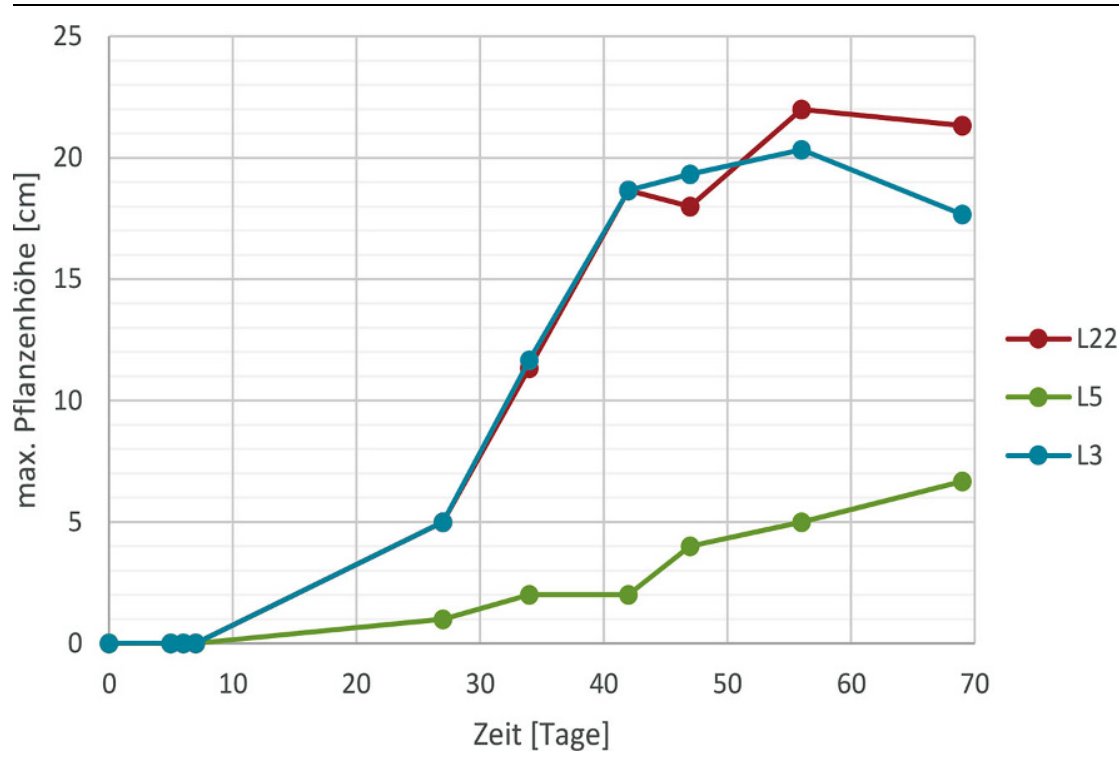

Abb. 5 Verlauf der Pflanzenhöhe von Lactuca sativa für L22, L3 und L5 $(n=3)$

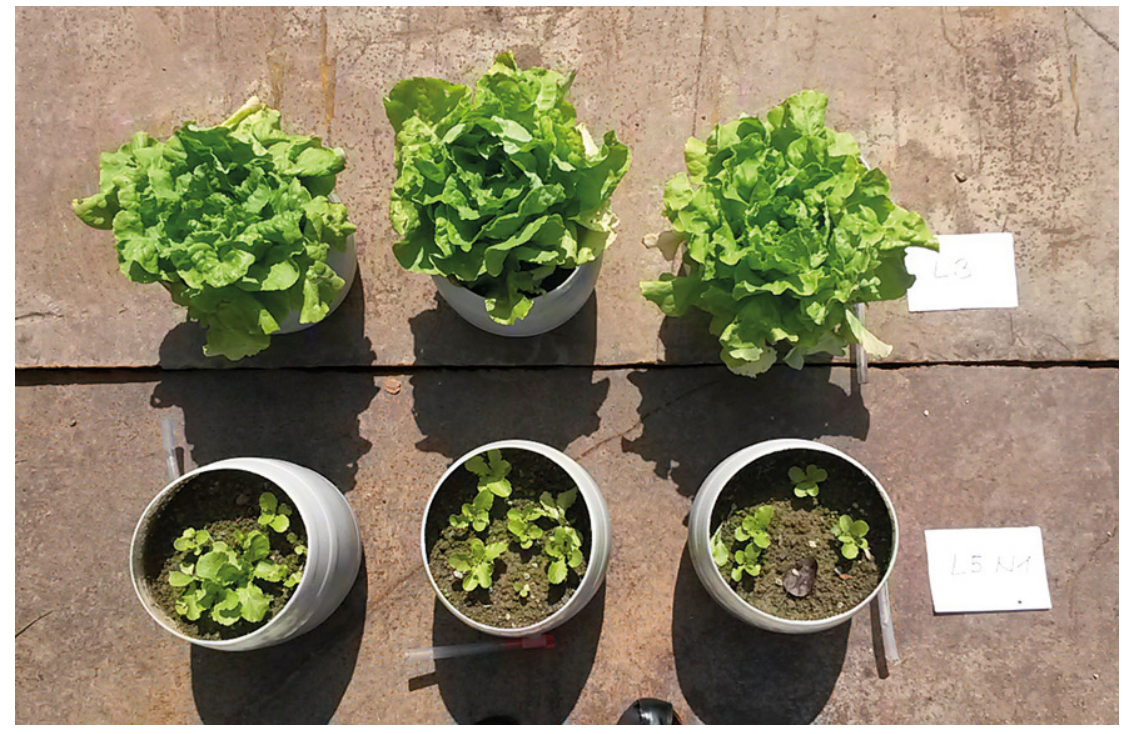

Abb. 6 Wachstumsunterschiede zwischen der Referenzprobe L3 und L5 bei Lactuca sativa nach $68 \mathrm{~d}$

und Ansätzen mit hohen Metallgehalten und teilweise keine messbaren Unterschiede. Lactuca sativa erwies sich als die ganzheitlich aussagekräftigste Zeigerpflanze. Beispielsweise zeigte sich für den Boden L5 mit den erhöhten Gehalten an $\mathrm{Cd}, \mathrm{Hg}$ und $\mathrm{Pb}$ ein eindeutiger Unterschied in Erntegewicht und Verlauf der Pflanzenhöhe (Abb. 5). Der Unterschied war auch mit bloßem Auge sichtbar (Abb. 6).

Es konnte bei allen untersuchten Metallen und Pflanzen zumindest bei einzelnen Bodenaushubmaterialien oder den daraus hergestellten Produkten ein Transfer vom Material in zeigt sich, dass nur der anthropogen Nibelastete Boden (L5N1) zu einer Überschreitung dieser Literaturwerte führt (Abb. 7).

\subsection{Großgefäßversuch}

Der Versuchsaufbau des Großgefäßtests war geeignet, um einen bedenklichen Transfer von Metallen in Bodenaushub bzw. daraus hergestellten Produkten, z. B. Komposterden, in das Sickerwasser von einem unbedenklichen $\mathrm{zu}$ unterscheiden.

Von den untersuchten Metallen zeigten sich bei sechs Substraten nur As bei einer Komposterde und $\mathrm{Ni}$ bei drei Komposterden als mobil und im Sickerwasser die Grenzwerte für Trinkwasser überschreitend. Bei Cu kam es im Vergleich zur Nullprobe zu erhöhten Werten im Sickerwasser, jedoch nicht zu Grenzwertüberschreitungen. Geogene Gehalte von $\mathrm{Cr}, \mathrm{Cu}$ und $\mathrm{Pb}$ führten $\mathrm{zu}$ keiner signifikanten Erhöhung der entsprechenden Metalle im Sickerwasser (Tab. 2).

Vergleicht man die Überschreitungen von Trinkwassergrenzwerten der Sickerwassergehalte der Komposterde L30 (zu 28\% aus Bodenaushub L5 bestehend) mit den Überschreitungen von BAWP-2017-Eluatgrenzwerten, Klasse A1, der Eluatgehalte der Komposterde und die Überschreitungen der BAWP-2017-Gesamtgehalte, Klasse A1, so zeigt sich, dass der Großgefäßtest von drei Grenzwertüberschreitungen der Gesamtgehalte, $\mathrm{Cd}$, Ni und $\mathrm{Pb}$ nur für Ni einen bedenklichen Transfer in das Sickerwasser nachweist, während beim Eluat keine BAWP-2017-Überschreitungen stattfanden (Tab. 3).

\section{Diskussion}

Die Praxis des stark reglementierten Bodenaushubmanagements in Österreich führt $\mathrm{zu}$ einer Bevorzugung der Deponierung vor der Verwertung, was sich bei hohem Aufkommen in einer Zunahme der Deponierungsrate niederschlägt. Zusätzlich sind die österreichischen Böden mit der sehr problematischen und auch zunehmenden Versiegelung konfrontiert. Die Abfallwirtschaft kann diesen beiden Entwicklungen auf der Seite der entstehenden Aushubmaterialien gegensteuern. Für landwirtschaftliche Böden wurden bereits fachliche, umweltgerechte und sinnvolle Rekultivierungsmaßnahmen mit Komposterden vorgeschlagen 


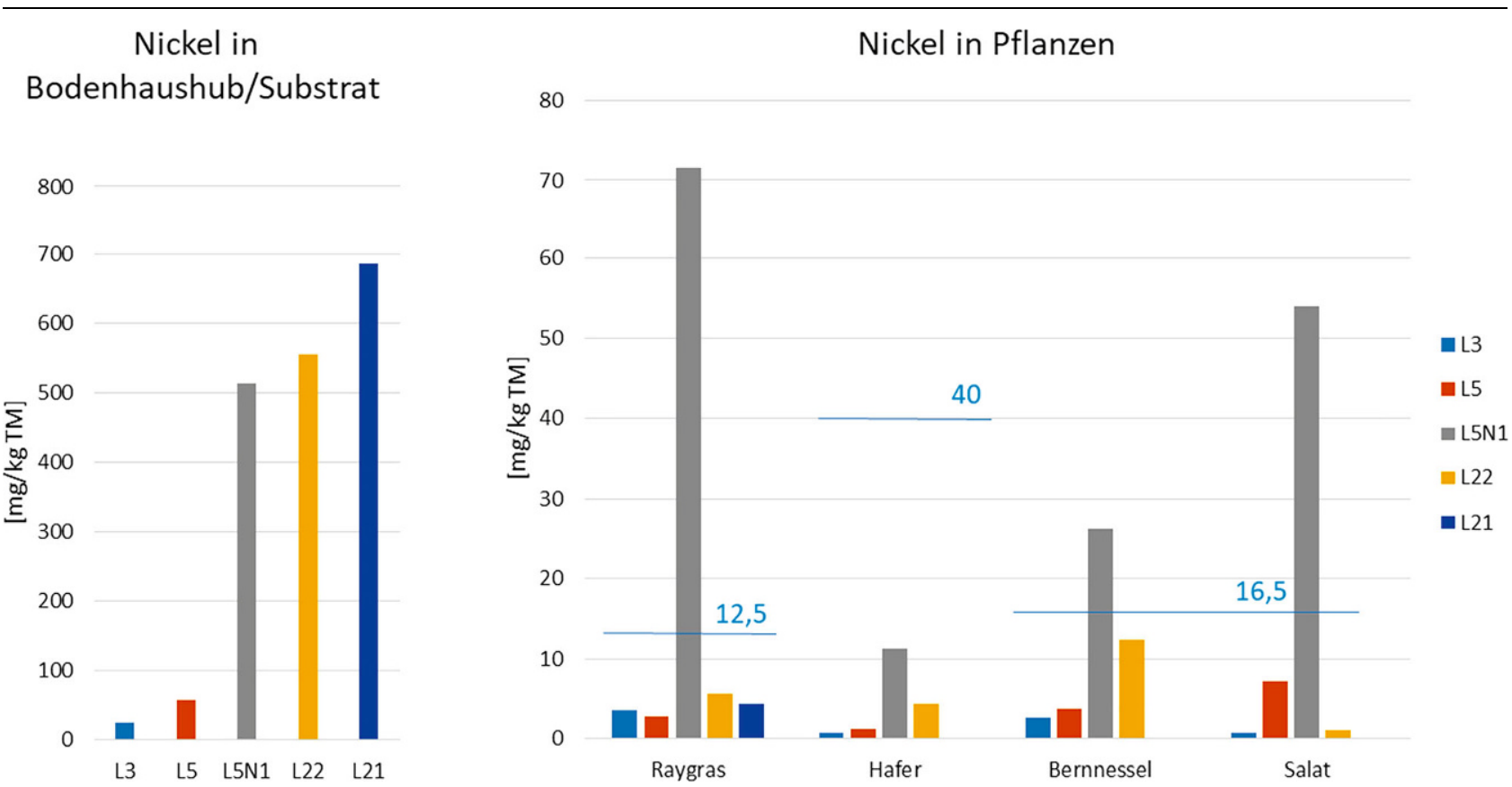

Abb. 7 Ni-Transfer von fünf Proben in vier Pflanzen im Kleingefäßtest ( $L 3$ Standardboden, $L 5$ mit Ni wenig belasteter Boden, L5N1 L5-Boden, der mit einer NiSO4 4 -Lösung versetzt wurde, L22 und L21 Komposterden mit geogener Ni-Belastung; blaue waagrechte Linie Literaturwerte von $\mathrm{Ni}$-Gehalten in Pflanzen, die auf $\mathrm{Ni}$-unbelasteten Böden gemessen wurden)

(BMLFUW 2012). Mit der vorliegenden Methode wird versucht, auch mit Metallen geogen belasteten, aber unbedenklichen Bodenaushub zu verwerten. Die hier vorgeschlagene Methodik ist ein alternativer Ansatz zum BAWP zur Beurteilung der Schadwirkung, der allerdings mit einem höheren Aufwand verbunden ist.

Würden geogen belastete, aber unbedenkliche Materialien nicht verwertet werden, müssten sie auf entsprechenden Deponien abgelagert werden. Damit würden die Funktionen des ursprünglichen Bodens in überwiegendem Maß dauerhaft verloren gehen. Diese Funktionen sind das Ergebnis von oft hunderten Jahren Bodenbildung, die weder technisch noch wirt- schaftlich von irgendeinem anderen Material in nur annähernd vergleichbarem Umfang wahrgenommen werden könnten.

\subsection{Die Qualitätssicherungsmethode}

Eine Unterscheidung in anthropogene und geogene Belastungen liegt mit der vorgeschlagenen Methode vor. Die Grundlage dafür ist die Annahme, dass anthropogene Belastungen kleinkörniger und oberflächlicher auftreten als geogene. Als Grenzen wurden aus methodischen Gründen die Korngröße von $2 \mathrm{~mm}$ und die Bodentiefe von $50 \mathrm{~cm}$ angenommen. Es handelt sich hierbei um Vereinfachungen, die möglichst nahe an der Praxis des Bodenaushubmanagements liegen sollen.

Eine Beurteilung von Materialien, die bereits ausgehoben vorliegen oder den daraus hergestellten Produkten, ist hinsichtlich einer Bestimmung auf geogen oder anthropogen naturgemäß nicht mehr durchführbar.

Auch geogene Belastungen können toxisch und bedenklich sein, weshalb bei der vorliegenden Methode zusätzlich zur Feststellung der geogenen Entstehung auch die Schadwirkung auf zweifache Weise beurteilt werden muss.

Die Schadwirkung von Metallen in Böden kann verschiedenartig bestimmt werden. Toxizitätstests für aquatische und terrestrische Systeme sind verfügbar, haben aber Nachteile. Sie zeigen

Tab. 2 Vergleich von Grenzwerten der TVO 2001 mit jenen im Sickerwasser eines Standardbodenaushubs (L3) ohne Belastungen und fünf Komposterden mit Metallbelastungen

\begin{tabular}{|c|c|c|c|c|c|c|c|c|}
\hline \multirow[t]{2}{*}{ Metalle $[\mu \mathrm{g} / \mathrm{l}]$} & & & L3 & L21 & L24 & L29 & L30 & L31 \\
\hline & $\begin{array}{l}\text { Grenzwerte } \\
\text { TV0 } 2001\end{array}$ & Gießwasser & Nullprobe & $\begin{array}{l}\text { Kompost- } \\
\text { erde A }\end{array}$ & $\begin{array}{l}\text { Kompost- } \\
\text { erde B }\end{array}$ & $\begin{array}{l}\text { Kompost- } \\
\text { erde L12 }\end{array}$ & $\begin{array}{l}\text { Kompost- } \\
\text { erde L5 }\end{array}$ & $\begin{array}{l}\text { Kompost- } \\
\text { erde C }\end{array}$ \\
\hline As & 10 & 5,5 & 5,8 & 7,7 & 7,7 & 8,8 & 9,2 & 15 \\
\hline$C d$ & 5 & $<0,1$ & $<0,1$ & $<0,1$ & $<0,1$ & $<0,1$ & $<0,1$ & 0,2 \\
\hline $\mathrm{Cr}$ & 50 & 2,6 & 2,2 & 2,7 & 2,5 & 2,7 & 2,7 & 2,6 \\
\hline $\mathrm{Cu}$ & 2000 & 1,3 & 15 & 15 & 22 & 26 & 27 & 19 \\
\hline $\mathrm{Hg}$ & 1 & $<0,1$ & $<0,1$ & $<0,1$ & $<0,1$ & $<0,1$ & $<0,1$ & $<0,1$ \\
\hline $\mathrm{Ni}$ & 20 & 1,4 & 11 & 14 & 24 & 15 & 21 & 39 \\
\hline$P b$ & 10 & $<0,1$ & $<0,1$ & $<0,1$ & $<0,1$ & $<0,1$ & $<0,1$ & $<0,1$ \\
\hline
\end{tabular}


Tab. 3 Vergleich der Ergebnisse der Untersuchungen der Komposterde L30, Gesamtgehalte, Eluat und Großgefäßtest, jeweils mit Grenzwerten

\begin{tabular}{|c|c|c|c|c|c|c|}
\hline \multirow[t]{3}{*}{ Metalle } & \multicolumn{2}{|l|}{ Gesamtgehalte } & \multicolumn{2}{|l|}{ Eluat } & \multicolumn{2}{|c|}{ Großgefäßetest } \\
\hline & BAWP 2017 A1 & L30 & BAWP 2017 A1 & L30 & TV0 2001 & L30 \\
\hline & $\begin{array}{l}\text { Grenzwerte } \\
\text { Gesamtgehalt } \\
{[\mathrm{mg} / \mathrm{kg} \mathrm{TM}]}\end{array}$ & $\begin{array}{l}\text { Komposterde L5 } \\
\text { Gesamtgehalt } \\
{[\mathrm{mg} / \mathrm{kg} \mathrm{TM}]}\end{array}$ & $\begin{array}{l}\text { Grenzwerte } \\
\text { Eluat } \\
{[\mathrm{mg} / \mathrm{kg} \mathrm{TM}]}\end{array}$ & $\begin{array}{l}\text { Komposterde L5 } \\
\text { Eluat } \\
\text { [mg/kg TM] }\end{array}$ & $\begin{array}{l}\text { Grenzwerte } \\
{[\mu \mathrm{g} / \mathrm{l}]}\end{array}$ & $\begin{array}{l}\text { Komposterde } L 5 \\
\text { Sickerwasser }[\mu \mathrm{g} / \mathrm{L}]\end{array}$ \\
\hline As & 20 & 7,45 & 0,3 & 0,11 & 10 & 9,2 \\
\hline$C d$ & 0,5 & 0,72 & 0,03 & 0,0029 & 5 & $<0,1$ \\
\hline $\mathrm{Cr}$ & 90 & 54,5 & 0,3 & 0,018 & 50 & 2,7 \\
\hline $\mathrm{Cu}$ & 60 & 46 & 0,6 & 0,14 & 2000 & 27 \\
\hline $\mathrm{Hg}$ & 0,5 & 0,49 & 0,01 & 0 & 1 & $<0,1$ \\
\hline $\mathrm{Ni}$ & 60 & 83 & 0,4 & 0,13 & 20 & 21 \\
\hline$P b$ & 100 & 165 & 0,3 & 0,13 & 10 & $<0,1$ \\
\hline
\end{tabular}

eingeschränkte Messbereiche, die über gesetzlich festgelegten Grenzwerten liegen (Moser und Römbke 2009), sie sind mit hohem Aufwand und Expertise verbunden (Conder und Lanno 2003) und haben oft hohe Streuungen (Wruss 2011). Als Alternative zur Bestimmung der Schadwirkung wurden bei der vorliegenden Methode der Transfer in Futter- und Lebensmittel sowie in das Sickerwasser und der Vergleich mit gesetzlich festgelegten Grenzwerten für Futter- und Lebensmittel sowie für Trinkwasser herangezogen.

Die Methode wurde an 12 Böden und 14 Bodensubstraten erprobt. Bei allen drei methodischen Schritten konnte anhand der Ergebnisse eine Unterscheidung in bedenkliche und unbedenkliche Materialien getroffen werden. Von 12 Böden waren zehn mit Metallen grenzwertüberschreitend belastet, wovon acht als anthropogen und zwei als geogen eingestuft wurden. Von fünf im Kleingefäßtest untersuchten Materialien waren vier mit Metallen grenzwertüberschreitend belastet, wovon zwei als bedenklich und zwei als unbedenklich eingestuft wurden. Von sechs im Großgefäßtest untersuchten Materialien waren fünf mit Metallen grenzwertüberschreitend belastet, wovon drei als bedenklich und zwei als unbedenklich eingestuft wurden.

Im Vergleich zum Kleingefäßtest zeigt der Großgefäßtest einen deutlich höheren Aufwand für die Durchführung. Um die Großgefäße mit den Substraten $\mathrm{zu}$ befüllen, bedarf es einiger Maschinen und es werden 3001 Bodensubstrat pro Ansatz benötigt, während der Kleingefäßtest manuell angesetzt werden kann und nur 101 Material benötigt. Jedoch wird der Großgefäßtest benötigt, da eine bessere Aussagekraft über das Sickerwasser erzielt werden kann, weil durch das größere Verhältnis von Randbereich zu Fläche weniger Kurzschlussströme auftreten können. Der Aufbau des „Bodens“ ist praxisnäher als beim Kleingefäßtest, da in den großen Gefäßen leichter mehrere Schichten (Unterboden und Oberboden) aufgebracht werden können. So können die Eigenschaften eines Substrats auf eine Weise analysiert werden, die seinem späteren Einsatz realitätsnäher entspricht.

\subsection{Bewertung der Ergebnisse am Beispiel Nickel}

In den getesteten Böden und Substraten gab es teilweise nur einzelne geogen bedingte Grenzwertüberschreitungen. Einige Materialien erfüllten beim Großteil der Parameter die Vorgaben des BAWP 2017 und würden entsprechend der Metallgehalte einer Verwertung zugeordnet werden können, wenn nicht ein einzelnes Metall in zu hoher Konzentration vorläge.

Am Beispiel von Ni sollen für Böden und daraus hergestellten Produkten mit überhöhten Gehalten folgende Aussagen zur Verwertung von Aushubmaterialien mit Grenzwerte des BAWP 2017 überschreitenden geogenen Gehalten festgehalten werden:

Die natürliche geogene Ni-Belastung in der steirischen Mur-Mürz-Furche ist langjährig bekannt. Der noch als natürlich einzustufende maximale $\mathrm{Ni}$ ckelgehalt steirischer Böden liegt bei $70 \mathrm{mg} / \mathrm{kg}$ TM und ist damit bereits höher als der BAWP-2017-Grenzwert der Qualitätsklassen A1 und A2 $(60 \mathrm{mg} / \mathrm{kg}$ TM) (Referat Boden- und Pflanzenanalytik 2015). Auch im Nahbereich einer potenziellen anthropogenen Quelle für $\mathrm{Ni}$, einem Stahlwerk in der Mur-MürzFurche, konnte kein Zusammenhang zwischen den Staub- und Ni-Dispositionen gefunden werden (Amt der Steiermärkischen Landesregierung 2003). Die Gesundheitsgefahren von Ni sind sogar bei direkter oraler Einnahme vernachlässigbar, nur bei Inhalation wird $\mathrm{Ni}$ als gefährlich eingestuft (European Food Safety Authority 2015). Es gibt für Ni weder einen Grenzwert in Lebensmitteln (Europäische Union 2006) noch in Futtermitteln (Europäische Union 2002). Unter diesen Gesichtspunkten konnten mit der vorliegenden Methode verschiedene Substrate mit erhöhten Gehalten an Ni in bedenkliche und unbedenkliche unterschieden werden.

\subsection{Grenzen der vorliegenden Arbeit}

Die Anzahl der untersuchten Materialien war limitiert, was eine statistische Auswertung nicht zuließ. Die dargestellten Ergebnisse sind nur eine Zusammenfassung der wichtigsten Erkenntnisse eines mehrjährigen Forschungsprojektes.

Danksagung Die Autoren bedanken sich bei der Österreichischen Forschungsförderungsgesellschaft $\mathrm{mbH}$ (FFG) und Poschacher Kompost für die finanzielle Unterstützung im Rahmen des Projektes ReSoil (FFG Nr. 848451) und bei Peter Liebhard und Anto Jelecevic von der Universität für Bodenkultur Wien, für die Zusammenarbeit.

Funding Open access funding provided by Montanuniversität Leoben.

Open Access Dieser Artikel wird unter der Creative Commons Namensnennung 4.0 International Lizenz (http:// creativecommons.org/licenses/by/4. 0/deed.de) veröffentlicht, welche die 
Nutzung, Vervielfältigung, Bearbeitung, Verbreitung und Wiedergabe in jeglichem Medium und Format erlaubt, sofern Sie den/die ursprünglichen Autor(en) und die Quelle ordnungsgemäß nennen, einen Link zur Creative Com- mons Lizenz beifügen und angeben, ob Änderungen vorgenommen wurden.

\section{Literatur}

Amt der Steiermärkischen Landesregierung (2017a): Landesumweltinformationssystem Steiermark (LUIS). www.umwelt.steiermark at. Gesehen 29. September 2019

Amt der Steiermärkischen Landesregierung (2017b): Geoinformationssystem des Landes Steiermark (GIS). http://gis2.stmk.gv.at. Gesehen 29. September 2019

Amt der Steiermärkischen Landesregierung (2003): Messung der Staub- und Schwermetalldeposition in Kapfenberg 1997 bis 2002. Fachabteilung 17C - Technische Umweltkontrolle und Sicherheitswesen, Graz

Amt der Steiermärkischen Landesregierung (2004): Regionale Hintergrundbelastung - Auswirkung auf Erzeugung und Verwertung von Komposten. Studie von Joanneum Research im Auftrag der FA 19D, Graz

Austrian Standards Institute (ASI) (2004): ÖNORM L1056: 2004 07 01 Probenahme von Dauergrünland (inklusive Parkanlagen, sowie Zier- und Sportrasen). Wien

Austrian Standards Institute (ASI) (2011) ÖNORM S2127:2011 1101 Grundlegende Charakterisierung von Abfallhaufen oder von festen Abfällen aus Behältnissen und Transportfahrzeugen. Wien

Austrian Standards Institute (ASI) (2016) ÖNORM EN ISO 17294-2: Wasserbeschaffenheit - Anwendung der induktiv gekoppelten Plasma-Massenspektrometrie (ICP-MS). Wien Austrian Standards Institute (2002): ÖNORM EN 13656: Charakterisierung von Abfällen Aufschluss mittels Mikrowellengerät mit einem Gemisch aus Fluorwasserstoffsäure (HF), Salpetersäure (HNO3) und Salzsäure $(\mathrm{HCl})$ für die anschließende Bestimmung der Elemente im Abfall. Wien

Austrian Standards Institute (2006): ÖNORM EN ISO 17294-2: Wasserbeschaffenheit - Anwendung der induktiv gekoppelten PlasmaMassenspektroskopie (ICP-MS). Wien

Brechlmacher, M. \& Wellacher, M. (2018): Katalog zu Bodenproben aus der Steiermark mi geogener Metallbelastung. In: Recy \& DepoTech 2018. Tagungsband zur 14. Recy \& DepoTechKonferenz 6.-9.11.2016. Leoben

Bundesgesetz (2001): Verordnung des Bundesministers für soziale Sicherheit und Generationen über die Qualität von Wasser für den menschlichen Gebrauch (Trinkwasserverordnung - TWV). BGBl. II Nr. 304/2001
Bundesgesetz (2008): Verordnung des Bundesministers für Land- und Forstwirtschaft, Umwelt und Wasserwirtschaft über Deponien - Deponieverordnung. BGBl II 39/2008

Bundesministerium für Land- und Forstwirtschaft, Umwelt und Wasserwirtschaft (BMLFUW) (2012): Richtlinien für die sachgerechte Bodenrekultivierung land- und forstwirtschaftlich genutzter Flächen. Wien

Bundesministerium für Land- und Forstwirtschaft, Umwelt und Wasserwirtschaft (BMLFUW) (2017): Bundes-Abfallwirtschaftsplan 2017 - Teil 1. Wien

Bundesministerium für Nachhaltigkeit und Tourismus (BMNT) (2019): Die Bestandsaufnahme der Abfallwirtschaft in Österreich - Stanahme der Abfallwirts
tusbericht 2019. Wien

Bundesministerium für Verkehr, Innovation und Technologie (BMVIT) (2011): Gewässerschutz an Straßen - RVS 04.04.11. Österreichische Forschungsgesellschaft Straße - Schiene Verkehr (FSV), Wien

Conder, J.M., Lanno, R.P. (2003): Lethal critical body residues as measures of $\mathrm{Cd}, \mathrm{Pb}$ and $\mathrm{Zn}$ bioavailability and toxicity in the earthworm Eisenia fetida. J Soils \& Sediments 3, 13-20

Drobnik, T., Greiner, L., Keller, A., Grêt-Regamy, A. (2018): Soil quality indicators - From soil functions to ecosystem services. Ecological Indicators, 94, 151-169

Europäische Union (2002): Richtlinie 2002/32/ EG des Europäischen Parlaments und des Rates vom 7. Mai 2002 über unerwünschte Stoffe in der Tierernährung

Europäische Union (2006): Verordnung Nr. 1881/ 2006 der Kommission vom 19. Dezember 2006 zur Festsetzung der Höchstgehalte für bestimmte Kontaminanten in Lebensmitteln. 2006/1881/EG

European Food Safety Authority (2015): Scientific Opinion on the risks to public health related to the presence of nickel in food and drinking water. ESFA, Italy, EFSA Journal 2015;13(2):4002 Fang, W., Yonghong, W., Liu, J. (2016): Comparative characterization of sewage sludge compost and soil: Heavy metal leaching characteristics. J.hazmat. 310, 1-10

Kabata-Pendias, A., Pendias, H. (2001): Trace elements in soils and plants. CRC Press, New York

Landwirtschaftliches Versuchszentrum Steiermark (LwVzSt) (1999): Bodenschutzbericht
1999 - Potentielle Kontaminationsflächen in der Steiermark. Land Steiermark, Rechtsabteilung 8, Graz

Moser, H., Römbke, J. (2009): Ecotoxicological Characterization of Waste - Results and Experiences of an International Ring Test. Springer Science+Business Media, New York

Österreichischer Wasser- und Abfallwirtschaftsverband (ÖWAV) (2014): ÖWAV-Arbeitsbehelf 44 - Herstellung von Komposterden (Mischungen aus Kompost und Bodenaushubmaterial). ÖWAV, Wien

Referat Boden- und Pflanzenanalytik (2015): Bodenschutzbericht 2015 - Bodenzustandsinventur Bezirk Liezen. Amt der Steiermärkischen Landesregierung Abteilung 10 Land- und Forstwirtschaft, Graz

Reinhofer M., Proske H., Pirkl, U., Kellerer-

Pirklbauer, A. (2004): Geogene Hintergrundbelastungen - Auswirkungen auf abfallwirtschaftliche Maßnahmen. Land Steiermark, Fachabteilung 19D, Graz, Eigenverlag des Landes Steiermark, Fachabteilung 19D

Tessier, A., Campbell, P., Bisson, M. (1979): Sequential Extraction Procedure for the Speciation of Particulate Trace Metals. Analytical Chemistry, 51(7), 844-851.

Umweltbundesamt (2017): Flächeninanspruchnahme in Österreich 2016. www.umweltbundes amt.at, Gesehen 29. September 2019

Wellacher, M., Pomberger, R., Vollprecht, D., Poschacher, F., Liebhard, P. \& Jelecevic, A. (2018): Verwertung von Aushubmaterialien. Tagungsbeitrag für Recy\&DepoTech 2018, 7.-9.11. 2018, Leoben

Wruss, K.W. (2011): Toxikologische Tests zur Risikominimierung bei Altlastensanierungsverfahren. Dissertation am Institut für nachhaltige Abfallwirtschaft und Entsorgungstechnik, Montanuniversität Leoben

Hinweis des Verlags Der Verlag bleibt in Hinblick auf geografische Zuordnungen und Gebietsbezeichnungen in veröffentlichten Karten und Institutsadressen neutral. 